Z Herz-Thorax- Gefäßchir 2008 · 22:110-118 DOI 10.1007/s00398-008-0623-7 Online publiziert: 25. März 2008 (c) Springer Medizin Verlag 2008

U. Boeken · P. Feindt

Klinik für Thorax- und Kardiovaskularchirurgie, Heinrich-Heine-Universität, Düsseldorf, BRD

\title{
Ist das SIRS/Sepsis-Syndrom in der Herzchirurgie Folge der extrakorporalen Zirkulation und damit unvermeidlich?
}

Viele Faktoren während der EKZ werden in Zusammenhang mit diesem Syndrom gebracht, zum einen materialabhängige Faktoren wie der Kontakt des Bluts mit fremden, nicht physiologischen Oberflächen, zum anderen materialunabhängige Faktoren wie das chirurgische Trauma. Um die sog. materialabhängigen Faktoren $\mathrm{zu}$ minimieren, werden gerade angesichts eines SIRS verstärkt sogenannte Off-pump-Verfahren diskutiert, also die Durchführung der Operation ohne HLM. Außerdem gewinnen vor diesem Hintergrund nicht zuletzt minimierende Verfahren der HLM, sog. MECC (miniaturized extracorporeal circulation)-Verfahren, zunehmend an Bedeutung.

Bei allem Nutzen einer Operation mit Hilfe der HLM müssen wir uns also stets auch fragen, welchen Schaden wir dem Patienten ggf. mit der EKZ zufügen.

\section{Definition und klinisches Bild des SIRS/Sepsis-Syndroms}

Zur einheitlichen Beschreibung des SIRS/ Sepsis-Syndroms und zur Abgrenzung von einer primären Sepsis wurden auf der ACCP/SCCM Consensus Conference des American College of Chest Physicians und der Society of Critical Care Medicine 1991 die Begriffe „systemic inflammatory response syndrome“ (SIRS) und „multiple organ dysfunction syndrome" (MODS) eingeführt [10].

Sepsis stellt die systemische Antwort auf eine Infektion dar, wohingegen SIRS die Reaktion auf inflammatorische Prozesse unabhängig von ihrer Genese ist.

Die klinische Manifestation eines SIRS ist durch das Auftreten von mindestens zwei der folgenden Symptome definiert:

1. Körpertemperatur $>38^{\circ} \mathrm{C}$ oder $<36^{\circ} \mathrm{C}$

2. Herzfrequenz $>90 / \mathrm{min}$

3. Atemfrequenz $>20 / \mathrm{min}$, bzw. Hyperventilation mit $\mathrm{PaCO}_{2}<32 \mathrm{mmHg}$

4. Leukozytenzahlen $>12000 / \mu$ l oder

$<4000 / \mu$ l oder $>10 \%$ stabkernige

Granulozyten im Differenzialblutbild

Es ist mittlerweile bekannt, dass inflammatorische Prozesse im Körper nicht nur mikrobiell, sondern auch toxisch, traumatisch, immunologisch und ischämisch ausgelöst werden können [10]. Diese Vorgänge dienen prinzipiell Inaktivierung und Beseitigung der stimulierenden Noxe und haben eigentlich die Einleitung reparativer Heilungsprozesse zum Ziel. Sie können allerdings durch Aktivierung humoraler und zellulärer Mediatorsysteme auch zu unkontrollierten Reaktionen mit einer Schädigung körpereigener Strukturen führen. Eine eingeschränkte Organfunktion kann daher Konsequenz einer systemischen inflammatorischen Reakti- 
on sein, die durch eine Beeinträchtigung der Mikrozirkulation gekennzeichnet ist. Grundlagen dieses Mechanismus sind in erster Linie Veränderungen des Vasotonus und der mikrovaskulären Permeabilität, mit dem Vollbild des Capillary-leakageSyndroms.

Nicht selten ist die Folge eines SIRS ein Multiorganversagen (MODS). Der Organismus reagiert dann uniform, d.h. unabhängig vom auslösenden Stimulus. Es folgen Minderperfusion und Ödembildung mit Funktionsausfall vitaler Organe. Durch Veränderungen von Vasotonus und Gefäßpermeabilität kommt es in der Lunge zur Ödembildung, zu Veränderungen des Ventilations-Perfusionsverhältnisses und zur Abnahme der Compliance [5]. Die daraus resultierende Hypoxämie verstärkt die bereits bestehenden peripheren Mikrozirkulationsstörungen zusätzlich. Eine generalisierte Vasodilatation und ein gesteigertes Herzzeitvolumen (HZV) kennzeichnen zunächst die sog. „hyperdyname Kreislaufsituation“. Es schließt sich eine hypodyname Phase mit Abfall des HZV und schwerem Schockzustand an; die Reagibilität der Gefäße auf Vasopressoren zur Aufrechterhaltung des Blutdrucks ist eingeschränkt. In dieser Phase der Erkrankung gelingt es häufig nicht mehr, eine suffiziente symptomatische Therapie einzuleiten. In vielen Fällen folgt bei primär unauffälligem mikrobiologischem Befund nun die sekundäre Keimbesiedlung der hypoxischen Organe und ggf. eine systemische Keimeinschwemmung (• Abb. 1).

\section{OPCAB, konventionelle ACB-OP, MECC}

Seit Mitte der 9oer Jahre werden koronare Bypassoperationen auch ohne Verwendung der extrakorporalen Zirkulation als Off-pump-coronary-artery-bypass (OPCAB)-Prozeduren vorgenommen, um die Morbidität der extrakorporalen Zirkulation, des kardioplegischen Herzstillstands und der transversalen Aortenklemmung zu vermeiden. Erste Vergleichsstudien zwischen der konventionellen Bypass- und der OPCAB-Chirurgie zeigten dabei eine Überlegenheit der OPCAB-Verfahren; diese waren jedoch überwiegend an Niedrigrisikopatienten vorge-
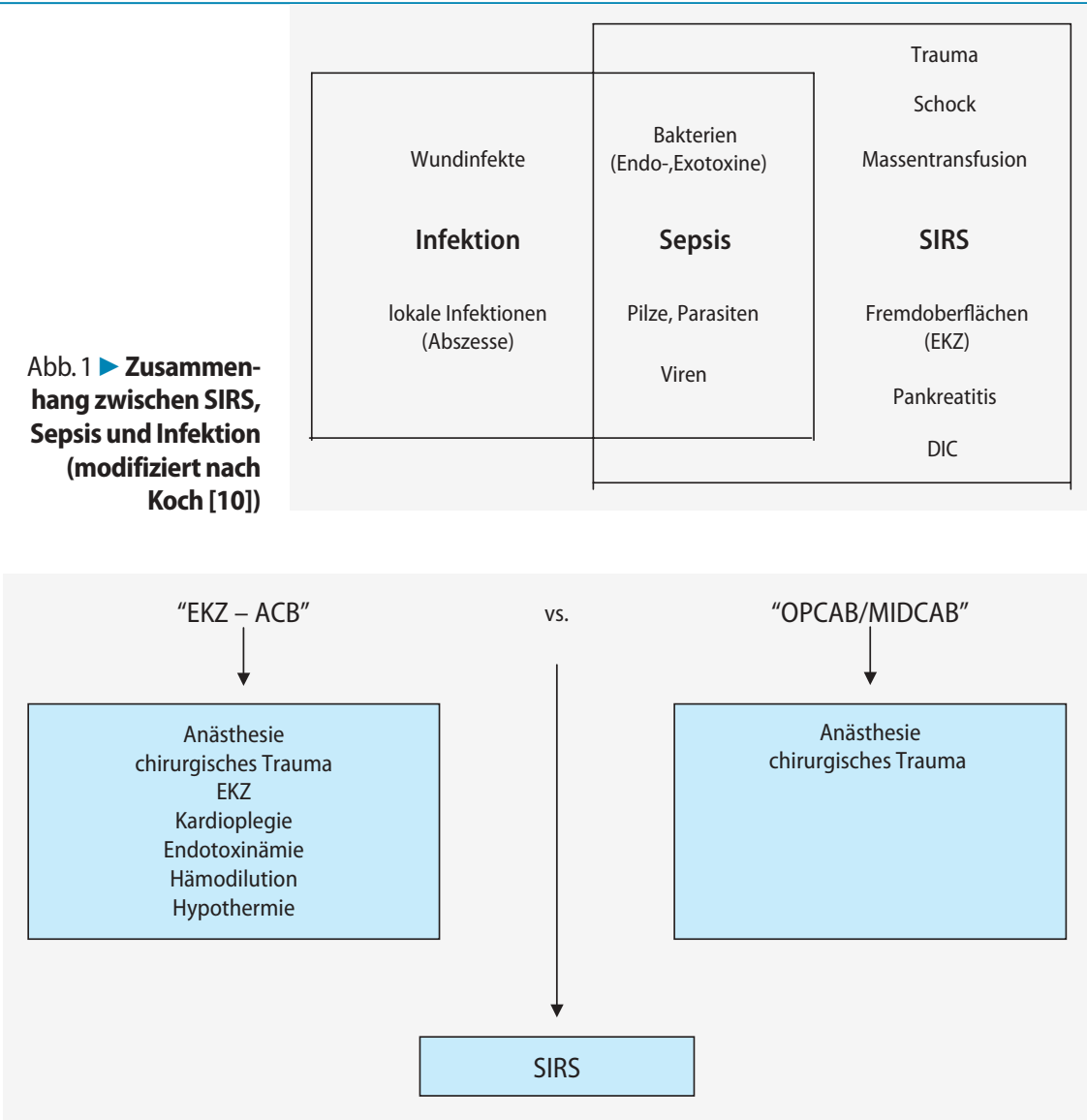

Abb. $2 \Delta$ Faktoren für die Entstehung eines SIRS - On-pump- vs. Off-pump-Verfahren

nommen worden, weshalb eine Verfälschung der Ergebnisse zugunsten der OPCAB-Chirurgie meist nicht ausgeschlossen werden konnte [20]. Bis heute liegt nun eine Vielzahl prospektiv-randomisierter Studien an unselektierten Kollektiven vor, die jedoch angesichts der jeweils niedrigen Patientenzahl und der geringen Inzidenz schwerer perioperativer Komplikationen das nötige Signifikanzniveau zugunsten des einen oder anderen Verfahrens in der Regel nicht erreichten. Auch in wenigen jüngst erschienenen Metaanalysen dieser randomisierten Studien fand sich meist kein signifikanter Unterschied bezüglich perioperativer Mortalität sowie Myokardinfarkt- und Schlaganfallrate. Eine Überlegenheit der OPCABChirurgie war hier jedoch bezüglich des Auftretens von Rethorakotomien, des perioperativen Blutverlusts, der Beatmungszeit, der Transfusionspflichtigkeit und folglich der Intensiv- und Krankenhausliegedauer sowie des Ressourcenverbrauchs erkennbar. Bis dato vorliegende große multizentrische Beobachtungsstu- dien belegen meist einen Vorteil der OPCAB-Chirurgie hinsichtlich perioperativer Mortalität, Myokardinfarkt- und Schlaganfallrate. Mittel- und langfristige Vergleichsuntersuchungen zu Anginafreiheit, Reinterventionsrate und Langzeitüberleben ergeben bislang keinen signifikanten Vorteil für das eine oder andere Verfahren [9].

Mehrere Studien konnten jedoch zeigen, dass die Inzidenz einer postoperativen, inflammatorischen Komplikation bei Patienten, die konventionell mit HLM operiert wurden, signifikant höher war [18]. Die Schlussfolgerung liegt nahe, dass allein die EKZ bereits einen Trigger für das Auslösen der inflammatorischen Kaskade darstellen kann ( $\bullet$ Abb. 2). Hieraus lässt sich allerdings nicht schließen, wie ausgeprägt die klinischen Veränderungen jedes einzelnen Patienten sein werden.

Unter dieser Annahme, dass nicht zuletzt die Fremdoberflächen eine entscheidende Rolle spielen, müssen in weiteren Studien auch die sog. MECC-Verfahren bezüglich inflammatorischer Pro- 
bleme analysiert werden. Hier liegen allerdings bisher noch keine validen Studien vor.

\section{Besonderheiten der extra- korporalen Zirkulation: Post-Perfusions-Syndrom}

Viele Operationen am Herzen sind auch heute nur mit Hilfe der EKZ durchführbar. Dieser kardiopulmonale Bypass kann ein systemisches Entzündungssyndrom hervorrufen [8]. Dieses sog. Post-Perfusions-Syndrom (PPS) endet in 2-10\% der Fälle im Vollbild eines SIRS. Viele Faktoren während der EKZ werden in Zusammenhang mit diesem Syndrom gebracht, zum einen materialabhängige Faktoren wie der Kontakt des Bluts mit fremden, nicht physiologischen Oberflächen, zum anderen materialunabhängige Faktoren wie das chirurgische Trauma [9], Ischämie-Reperfusionsschäden der Organe (z. B. durch Ausschluss der Lunge aus der Zirkulation während des totalen Bypass), Veränderungen der Körpertemperatur und Freisetzung von Endotoxin. Diese Mechanismen verursachen bei jedem Patienten eine individuelle Immunantwort mit Komplementaktivierung, $\mathrm{Zy}$ tokinfreisetzung, Leukozytenaktivierung inklusive Expression von Adhäsionsmolekülen und das Freisetzen von Sauerstoffradikalen, Arachidonsäuremetaboliten, plättchenaktivierendem Faktor (PAF), Stickstoffoxid (NO) und Endothelin [27]. Für jeden einzelnen Faktor wurden in prospektiven Studien erhöhte Spiegel nach komplikationslos verlaufener EKZ nachgewiesen, ohne dass bisher eine Korrelation zu klinischen Veränderungen der Patienten aufgezeigt werden konnte. Erst wenn diese messbaren Veränderungen mit klinischen Auffälligkeiten (erhöhter Volumenbedarf, Katecholaminpflichtigkeit, niedriger peripherer Gefäßwiderstand, interstitielles Lungenödem) einhergehen, spricht man von einem PPS. Dieses PPS ist in der Regel selbstlimitierend (24 bis $48 \mathrm{~h}$ ), es mündet allerdings bei einigen Patienten in ein SIRS, wobei die konkreten Ursachen hierfür nicht bekannt sind. Zur besseren Beurteilung möglicher therapeutischer Interventionsmöglichkeiten soll im Folgenden ein kurzer Überblick über die wichtigen Kaskaden der systemischen

Tabelle 1

Einteilung der wichtigsten bekannten Adhäsionsmoleküle [3]

Selektine (L-: Leukozyten; E-: Endothelzellen; P-: Thrombozyten)
GMP-140 (Endothelzellen)
MEL-14/LAM-1 (PMN)
ELAM-1 (Endothelzellen)
Integrine (neutrophile Granulozyten)
LFA-1
MAC-1
VLA-4
P150, 95
Immunglobulin-Superfamilie (Endothelzellen)
ICAM-1
ICAM-2
ICAM-3
VCAM-1,-2

inflammatorischen Reaktion gegeben werden.

\section{Komplementaktivierung}

Das Komplementsystem wird während EKZ sowohl über den klassischen als auch den alternativen Weg aktiviert. Der Kontakt des Bluts mit den Fremdoberflächen induziert den alternativen Weg mit Bildung von $\mathrm{C}_{3} \mathrm{a}$ und $\mathrm{C}_{5} \mathrm{a}$, wogegen die $\mathrm{He}$ parinantagonisierung mit Protamin zum klassischen Weg führt, verbunden mit einem Anstieg von $\mathrm{C}_{4}$ a und später $\mathrm{C}_{3} \mathrm{a}$. $\mathrm{C}_{3} \mathrm{a}$ und $\mathrm{C}_{5}$ a führen ihrerseits zur Histaminfreisetzung, steigern die Gefäßpermeabilität und stimulieren die Leukozyten zur Freisetzung von $\mathrm{O}_{2}$-Radikalen. $\mathrm{C}_{3}$ a ist ein potenter Stimulator der Plättchenaggregation, wohingegen C5a zur Aggregation von neutrophilen Granulozyten und deren Adhärenz am Endothel führt.

\section{Leukozyten und Adhäsions- moleküle}

Die EKZ und die oben beschriebene Komplementaktivierung können zur Aktivierung von neutrophilen Granulozyten (PMN) und Endothelzellen führen. Hierdurch kommt es zu einer vorübergehenden Adhäsion der Leukozyten an das Endothel. Dabei spielen die Begriffe des "Rollings" und „Stickings" an der Gefäßwand sowie der Diapedese der PMN in das umgebende Gewebe eine entscheidende Rolle [3]. Diese Interaktionen werden vermittelt durch Oberflächenmoleküle auf PMN und Endothelzellen [1,3]. Man teilt diese sogenannten Adhäsionsmoleküle in drei Kategorien ein:

1) Integrin-Rezeptoren der Leukozyten, die mit Liganden der Endothelzelle reagieren,

2) Selectin-Rezeptoren, die auf beiden Zellen exprimiert werden, und

3) Immunglobulin-Superfamilie (nur auf Endothelzellen) [25].

- Tabelle 1 zeigt die wichtigsten heute bekannten Adhäsionsmoleküle. Wie wir mittlerweile wissen, führt die Induktion spezifischer Adhäsionsmoleküle durch verschiedene inflammatorische Stimuli (IL-1, IL-4, Interferon- $\gamma$, Histamin, Leukotriene) in Verbindung mit der Hochregulation spezifischer Adhäsionsrezeptoren auf den Leukozyten zur selektiven Expression bestimmter Leukozyten-Subpopulationen. Die daraufhin folgende Infiltration (Diapedese) des jeweiligen Organs durch zirkulierende Leukozyten stellt sicherlich einen grundlegenden Pathomechanismus bei SIRS/Sepsis, Transplantatabstoßung und Ischämie/Reperfusionsschaden dar. Demzufolge ist die Hemmung der Leukozytenadhäsion an das Endothel ein geeignet erscheinender Therapieansatz bei inflammatorischen und immunologischen Dysregulationen. 
Z Herz-Thorax- Gefäßchir 2008 · 22:110-118

DOI 10.1007/s00398-008-0623-7

C) Springer Medizin Verlag 2008

\section{U. Boeken · P. Feindt}

\section{Ist das SIRS/Sepsis-Syndrom in der Herzchirurgie Folge der extrakorporalen Zirkulation und damit unvermeidlich?}

\section{Zusammenfassung}

Hintergrund: Die Anwendung der extrakorporalen Zirkulation (EKZ) im Rahmen kardiochirurgischer Operationen führt zu einer ausgeprägten inflammatorischen Reaktion, die jedoch nur bei einem geringen Teil der betroffenen Patienten zu klinischen Komplikationen und erhöhter Letalität führt („eskalierendes SIRS”). Innerhalb der aktuell vorliegenden Literatur gibt es lediglich wenige, unzureichende Angaben über Veränderungen des Immunsystems bei Patienten, die in der frühen postoperativen Phase ein schweres SIRS (systemic inflammatory response syndrome) mit begleitenden Organinsuffizienzen entwickeln.

Methoden: Nach Durchsicht der grundlegenden Literatur sollen die Abläufe und Mediatorsysteme im Rahmen eines SIRS nach EKZ zusammenfassend dargestellt werden. Insbesondere die Unterschiede zwischen On-pump- und Off-Pump-Verfahren in der Herzchirurgie werden diskutiert, mit besonderer Berücksichtigung von minimierten, extrakorporalen Systemen (miniaturized extracorporeal circulation = MECC). Darüber hinaus erläutern wir mögliche Therapien bei inflammatorischen Komplikationen.

Ergebnisse: Der Kontakt des Bluts mit Fremdoberflächen (EKZ) sowie die Endotoxinfreisetzung aus dem Darm führen zur Aktivierung von Komplementsystem und Zytokinkaskade. Nachgeschaltete Kaskadensysteme induzieren anschließend die Aktivierung verschiedener Zellreihen. Die resultierende Organdysfunktion führt zum klinischen Bild eines Post-Perfusions-Syndroms (PPS), das in 2-10\% der Fälle in ein SIRS übergeht, wobei die auslösenden Mechanismen bei den betroffenen Patienten bis heute unbekannt sind.
Therapieansätze zur Abschwächung dieser Reaktionen beinhalten u.a. die Gabe von Kortikosteroiden, Aprotinin und Antioxidantien sowie die Verwendung Heparin-beschichteter EKZ-Systeme, intraoperativer Ultra- und Leukozytenfiltration. Fazit: Das eskalierende SIRS nach herzchirurgischen Operationen stellt ein uneinheitliches immunologisches Geschehen dar, welches sowohl pro- als auch anti-inflammatorische Komponenten beinhaltet. Bei einer Inzidenz von ca. $20 \%$ für ein PPS nach EKZ ist weiter unklar, ob die EKZ allein ein Auslöser ist und warum es bei ausgewählten Patienten zu einer Aggravierung kommt. Die frühe Erkennung solcher "patients at risk" ist der momentan einzige sinnvolle Ansatz.

\section{Schlüsselwörter}

SIRS, Sepsis · kardiopulmonaler Bypass . extrakorporale Zirkulation · Zytokine

\section{Is a SIRS/sepsis syndrome after cardiac surgery a consequence of extracorporeal circulation?}

\section{Abstract}

Background: The inflammatory response to cardiopulmonary bypass (CPB) is the product of a complex interplay of humoral and cellular components. Recent study of this SIRS (systemic inflammatory response syndrome)/sepsis syndrome in cardiac surgery has improved our understanding of the involvement of this inflammatory cascade in perioperative injury. However, the exact mechanisms of this complex response remain to be fully determined.

Methods: We reviewed the basic literature on the inflammatory response, especially following CPB, to define current knowledge on the possible pathways and mediators involved. We particularly focussed on differ- ences between "on- and off-pump" cardiac surgery and on therapeutic strategies. Results: Contact activation cascades, the complement system, and cytokines interact in such a way as to propagate their own cascades and to activate the cellular elements. The resulting organ dysfunction produces the clinical picture of a "post-perfusion syndrome" or a SIRS. It is still unknown why there is an escalating inflammatory reaction in some patients. Strategies to attenuate this response include the administration of corticosteroids, aprotinin, and antioxidants, as well as the modification of equipment by the use of heparin-coated CPB circuits, leukocyte depletion, and ultrafiltration.
Conclusions: A better understanding of the inflammatory response and the development of new therapeutic approaches can lead to improved patient outcome, perhaps after early detection of "patients at risk". Further studies are necessary to consider the individual immunologic competence of each patient and to decrease the mortality of SIRS/sepsis. However, extracorporeal circulation alone may be a trigger for an inflammatory complication.

\section{Keywords}

SIRS - sepsis - cardiopulmonary bypass . extracorporeal circulation · cytokines 


\section{Sauerstoffradikale}

Der Leukozytenaktivierung folgt u.a. die Freisetzung von toxischen Sauerstoff $\left(\mathrm{O}_{2}\right)$ Radikalen. Diese reagieren mit den Membranlipiden und führen so zu einer gesteigerten Membranpermeabilität mit Ausbildung eines Ischämie-Reperfusionsschadens und nachfolgender Beeinträchtigung der kardialen und pulmonalen Funktion. In verschiedenen Studien konnte eine Korrelation zwischen dem Grad der Komplementaktivierung und der Menge zirkulierender $\mathrm{O}_{2}$-Radikale nachgewiesen werden. $\mathrm{O}_{2}$-Radikale führen zudem zu einer Abnahme des verfügbaren $\mathrm{NO}$, woraus wiederum Vasospasmus und Thrombose nach Reperfusion eines ischämischen Herzens resultieren können.

\section{Arachidonsäuremetabolite}

Durch die Freisetzung von Arachidonsäure kommt es zur Bildung von Thromboxan $\mathrm{A}_{2}(\mathrm{Tx})$, Prostaglandinen und Leukotrienen, hauptsächlich auf der Zellmembran der PMN.

Thromboxan hat starke vasokonstriktive Eigenschaften und führt $\mathrm{zu}$ einer Plättchenaggregation. Prostaglandine (PG) hingegen ( $\mathrm{PGE}_{1}, \mathrm{PGE}_{2}$ und Prostacyclin $=\mathrm{PGI}_{2}$ ) sind aufgrund ihrer vasodilatierenden und plättchenaggregationshemmenden Wirkung die wichtigsten Gegenspieler des Thromboxans, wobei die Relation (ratio) von $\mathrm{PGI}_{2} \mathrm{zu}_{\mathrm{TxA}}$ für viele endotheliale und gerinnungsphysiologische Abläufe von großer Bedeutung zu sein scheint. Leukotriene sind potente Chemokine, die zu einer Erhöhung der Gefäßpermeabilität führen.

\section{Endotoxin}

Endotoxin ist ein wichtiger Aktivator der inflammatorischen Kaskade. Während der EKZ gibt es viele Möglichkeiten einer Endotoxin-Freisetzung, wobei der Darm sicherlich eine entscheidende Rolle spielt. Hier kann es zu einer Permeabilitätssteigerung durch Endothelischämie nach mesenterialer Vasokonstriktion kommen. Endotoxin führt über den alternativen Weg u.a. zur Aktivierung des Komplementsystems und zur Freisetzung von Zytokinen einschließlich TNF- $\alpha$.

\section{Zytokine}

Neben Endotoxin sind auch Ischämie sowie Komplementaktivierung Stimuli der Zytokinfreisetzung [19]. Während der EKZ kommt es insbesondere zu erhöhten Interleukin(IL)-6- und IL-8-Spiegeln. Diese proinflammatorischen Zytokine führen ebenso wie TNF-a [16] zu einer myokardialen Dysfunktion verbunden mit hämodynamischer Instabilität, wobei direkte hämodynamische Effekte bisher nicht nachgewiesen werden konnten. Im Gegensatz hierzu kommt es unter den Bedingungen der EKZ auch zur Sekretion des antiinflammatorischen IL-10, welches wiederum die Produktion proinflammatorischer Zytokine hemmt. Die Ausprägung einer inflammatorischen Antwort nach EKZ ist somit ganz entscheidend vom Verhältnis zwischen pro- und antiinflammatorischen Zytokinen abhängig.

\section{Plättchenaktivierender Faktor}

Plättchenaktivierender Faktor (PAF) ist ein Phospholipid und wird von vielen Zellen synthetisiert, so z.B. von Thrombozyten, Endothelzellen und Myozyten. Neben der plättchenaggregierenden und -aktivierenden Wirkung kommt es durch PAF auch zu einer Aktivierung von PMN, infolgedessen es eine entscheidende Rolle in der Entwicklung eines Ischämie-Reperfusionsschadens von Myokard und Lunge spielt. PAF-Rezeptoren werden ebenfalls auf Monozyten und Endothelzellen exprimiert, woraus sich die zentrale Bedeutung des PAF in der inflammatorischen Kaskade erklärt.

\section{Stickstoffoxid}

Stickstoffoxid (NO) ist einer der Hauptregulatoren des Gefäßtonus (endothelium derived relaxing factor $=\mathrm{EDRF}$ ) und damit des Blutflusses. Aufgrund einer Hemmung von Thrombozytenaggregation und Leukozytenadhäsion reduziert NO Thrombenbildung und Leukozytenaktivierung. Durch Konversion der Aminosäure L-Arginin zu L-Citrullin durch die NO-Synthase (NOS) kommt es zur Bildung von NO in den Endothelzellen. Mittels Endotoxin oder Zytokine kann es zu einer gesteigerten Synthese von NOS kommen, das vermehrt anfallende NO führt $\mathrm{zu}$ Vasodilatation und den hieraus resultierenden Konsequenzen. NOS-Bildung kann durch Kortikosteroide unspezifisch verringert werden. Verminderte NO-Bildung hingegen führt zu koronaren Vasospasmen, Plättchenaggregation und pulmonaler Hypertonie.

\section{Endothelin}

Endothelin-1 (ET-1) besteht aus 21 Aminosäuren und wird vom Endothel freigesetzt. Es ist der bisher stärkste bekannte endogene Vasokonstriktor und maßgeblich an der Regulation des arteriellen Blutdrucks beteiligt [24]. Die gemessenen ET1-Spiegel korrelieren mit der Dauer der EKZ [26]. Produktion und Abbau des ET1 finden hauptsächlich in der Lunge statt. Die Intensität der Vasokonstriktion und somit das Auftreten ischämischer Ereignisse sind ganz entscheidend vom Verhältnis des ET-1-Spiegels zu NO und $\mathrm{TxA}_{2}$ abhängig [28]. Sowohl Koronarspasmen nach aortokoronarer Bypassoperation, als auch intestinale Ischämien nach EKZ stehen sicherlich in engem Zusammenhang mit der ET-1-Produktion.

- Abb. 3 fasst die oben beschriebenen Kaskaden im Rahmen einer systemischen Inflammation während bzw. nach EKZ zusammen. Wichtiges Kriterium der ablaufenden komplexen Reaktionen ist dabei die zeitliche Dynamik: In der Frühphase lassen sich v.a. ansteigende Konzentrationen der proinflammatorischen Zytokine IL-1, IL-6 und IL-8 sowie TNF- $\alpha$ nachweisen, im Sinne eines hyperinflammatorischen Zustands des Organismus. Dieser Phase folgt eine sog. „Immunlücke“ bzw. „Immunparalyse“, die durch die antiinflammatorischen Substanzen IL-4, IL-10 und IL-13 gekennzeichnet sind und den Patienten für infektiöse Komplikationen prädisponieren.

\section{Einfluss der EKZ auf spezielle inflammatorische Abläufe}

Erhöhte C3a-Spiegel während der EKZ wurden bereits von zahlreichen Autoren beschrieben [2] sowie mit Dauer der EKZ und Art des Oxygenators korreliert. C3aSpiegel und der terminale Komplement- 


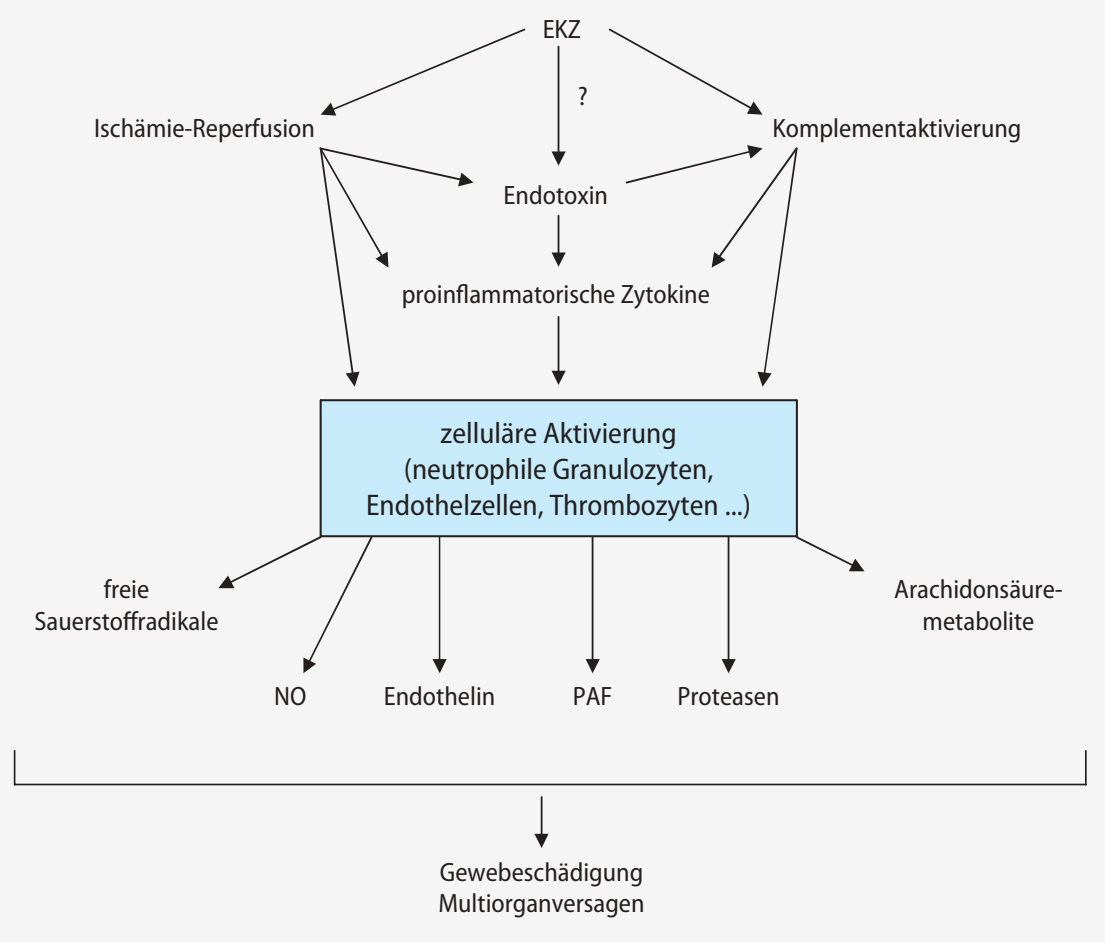

Abb. $3 \Delta$ Schematischer Überblick der inflammatorischen Antwort nach EKZ [27]

komplex sind jedoch bei größeren Operationen oder Herzoperationen ohne EKZ nicht erhöht [14], weshalb sowohl die oben beschriebene Kontaktaktivierung als auch die Heparin-Protamin-Reaktion aller Wahrscheinlichkeit nach die hauptsächlichen Triggermechanismen der Komplementkaskade bei Operationen mit EKZ sind. Aufgrund der schnellen Bindung an PMN ist die Bestimmung des $\mathrm{C}_{5} \mathrm{a}$ häufig schwieriger. Die klinische Relevanz einer Komplementaktivierung nach EKZ ist bisher allerdings nicht eindeutig geklärt. Verschiedene Studien konnten eine Korrelation zur postoperativen Morbidität nachweisen; so ging mit erhöhten $\mathrm{C}_{3} \mathrm{a}$ Spiegeln 3 Stunden nach EKZ signifikant häufiger ein komplizierter postoperativer Verlauf einher [17]. Dementgegen konnten andere Gruppen [27] keine Korrelation zwischen Komplementaktivierung und pulmonalen oder kardialen Komplikationen aufzeigen. Diese Unterschiede sind am ehesten durch die komplexe Natur der inflammatorischen Reaktion erklärbar, wobei das Komplementsystem nur einer von verschiedenen, durch die EKZ getriggerten Faktoren ist.

Eine erhöhte Expression von Adhäsionsmolekülen und die daraus resultierende Aktivierung von neutrophilen Granulozyten stellt einen entscheidenden Faktor in der Ausbildung von perioperativem Capillary-leakage-Syndrom und myokardialem Ischämie-Reperfusionsschaden dar [7]. Es konnte darüber hinaus nachgewiesen werden, dass eine Blockade der Adhäsionsmoleküle vor Reperfusion das Risiko einer myokardialen Insuffizienz signifikant verringert [7]. Obwohl bei manchen Patienten die Menge der zirkulierenden Adhäsionsmoleküle während elektiven Operationen mit EKZ nicht deutlich erhöht ist, kommt es in jedem Fall zu einer gesteigerten Expression von Adhäsionsmolekülen auf den Leukozyten unmittelbar nach EKZ-Beginn und nach Öffnen der Aortenklemme.

Der Anfall freier Sauerstoffradikale während und nach EKZ ist abhängig von vielen Faktoren, so beispielsweise dem Grad der Komplementaktivierung, der Art des verwendeten Oxygenators, dem $\mathrm{O}_{2}$-Gehalt von Einatemluft und Blut sowie der Bluttemperatur. Toxische $\mathrm{O}_{2}$-Radikale $\left(\mathrm{O}_{2}^{-}, \mathrm{H}_{2} \mathrm{O}_{2}, \mathrm{OH}^{-}, \mathrm{HOCl}\right)$ werden nach Leukozytendiapedese in umliegende Gewebe und dortiger Degranulation frei, wobei dieser Prozess insbesondere in Anwesenheit von Endotoxin abläuft [15]. Die toxische Wirkung resultiert zum einen aus der oben beschriebenen Peroxidation der Membranlipide und Nukleinsäuren, zum anderen aus der Inaktivierung physiolo- gischer Inhibitoren der proteolytischen Enzyme, die von neutrophilen Granulozyten freigesetzt werden. $\mathrm{OH}^{-}$führt sogar zu einer direkten Aktivierung von $\mathrm{C}_{5} \mathrm{zu}$ C5a, was im Sinne einer positiven Rückkopplung eine Verstärkung der inflammatorischen Reaktion bewirkt. Diese Wirkmechanismen und Interaktionen der $\mathrm{O}_{2}$-Radikale konnten bereits in vielen Studien nachgewiesen werden; nach unseren Erkenntnissen gibt es jedoch bisher keine Studie, die den Einfluss dieser Substanzen auf den postoperativen Verlauf nach EKZ eindeutig demonstrieren konnte.

Eine gesteigerte Synthese der Arachidonsäuremetabolite konnte in septischem und experimentellem Endotoxin-Schock nachgewiesen werden [7]. In Analogie zu tierexperimentellen Befunden fand sich auch bei allgemeinchirurgischen Patienten im hyperdynamen septischen Schock ein Überwiegen der Prostazyklinbildung, während bei der hypodynamen Form die Thromboxansynthese dominierte. Die Effekte von Thromboxan $\mathrm{A}_{2}$ $\left(\mathrm{TxA}_{2}\right)$ (Vasokonstriktion, Plättchenaggregation) halten nach EKZ zwar nur für einige Minuten an, in Tiermodellen konnten allerdings dennoch hierdurch bedingte myokardiale Dysfunktion und pulmonale Hypertonie nach EKZ provoziert werden. Prostaglandine wirken in diesem Zusammenhang antagonistisch zum $\mathrm{TxA}_{2}$, wobei sogar eine direkte Hemmung der $\mathrm{TxA}_{2}$-Freisetzung durch PGE1 beschrieben wurde [15].

Bezüglich des Endotoxins sind die Ergebnisse der vorliegenden Studien ausgesprochen variabel. In der Annahme, dass eine gesteigerte Permeabilität der gastrointestinalen Mukosa aufgrund passagerer Hypoxien die Ursache für einen erhöhten Anfall von Endotoxin ist, wurde von vielen Untersuchern der $\mathrm{pH}$ in der Mukosa gemessen. Die erwartete Azidose konnte während EKZ beobachtet werden [12], einige Studien aber zeigten, dass Endotoxin trotz unverändertem oder sogar alkalotischem $\mathrm{pH}$ vermehrt anfiel. Unseren Erachtens ist zwar bekannt, dass Endotoxin zu Komplementaktivierung und Freisetzung von Interleukinen führt [16], es ist aber bisher nicht gelungen, eine Korrelation zwischen Endotoxin-Spiegeln und Patienten-„,Outcome“ herzustellen. 
Mit prospektiven Untersuchungen konnte in der Vergangenheit gezeigt werden, dass einige Zytokine (IL-6, IL-8) und TNF- $\alpha$ zu myokardialer Dysfunktion und hämodynamischer Instabilität führen [2]. In verschiedenen Studien wurde die Zytokin-Freisetzung und deren zeitlicher Verlauf als eine Reaktion auf die EKZ nachgewiesen: Nach Beginn der EKZ kommt es rasch zum Anstieg von IL-1, IL-6, IL-8 und TNF- $a$. Es ist bis heute allerdings weitgehend unklar, von welchen Zellen diese Zytokine nach EKZ in vivo freigesetzt werden. In Zellkulturen konnte dies für neutrophile Granulozyten, Makrophagen und Endothelzellen gezeigt werden. Zur klinischen Relevanz der Zytokin-Antwort nach EKZ existieren extrem konträre Ergebnisse: Erhöhte Spiegel der proinflammatorischen Interleukine korrelieren in einigen Studien mit erhöhter Inzidenz von Multiorganversagen und perioperativer Letalität, in anderen Studien zeigt sich keine klinische Konsequenz der unterschiedlichen Zytokin-Antwort.

Während und nach EKZ gilt es heute als gesichert, dass es generell zu einer gesteigerten NO-Produktion kommt, was neben den oben beschriebenen Mechanismen durch Heparin noch verstärkt wird. Proinflammatorische Zytokine führen durch Induktion der NOS ebenfalls zu gesteigerter NO-Produktion. Erhöhte NOSSpiegel konnten im Lungengewebe von Patienten nach EKZ gemessen werden. Die Verabreichung von NO kann den Ischämie-Reperfusionsschaden reduzieren und die myokardiale Funktion verbessern [27]. In allen bekannten Studien werden die gemessenen NO-Spiegel allerdings nicht im Zusammenhang mit den übrigen Kaskaden einer inflammatorischen Reaktion gesehen, weshalb die Bedeutung des NO beim SIRS/Sepsis-Syndrom bislang nicht geklärt ist.

Eine signifikant erhöhte Freisetzung von ET-1 konnte bei Patienten nach Myokardrevakularisation mittels EKZ nachgewiesen werden [26]. Es ließ sich weiterhin zeigen, dass dieses ET-1 nicht vom Koronarendothel, sondern in der Lungenstrombahn produziert wird. Aufgrund der vasokonstriktiven Eigenschaften des ET-1 können Myokardischämien verursacht werden. Der Gefäßtonus jedes einzelnen Patienten ist allerdings abhängig vom Ver- hältnis des ET-1 zu TxA $\mathrm{A}_{2}$ und NO. Da $\mathrm{TxA}_{2}$ selbst zur ET-1-Freisetzung führt, ist das Risiko einer Minderperfusion bei $\mathrm{Pa}$ tienten mit erhöhtem $\mathrm{TxA}_{2}$ - und gleichzeitig verringerten NO-Spiegel (z.B Hemmung der NOS durch Kortikosteroide) am größten, wohingegen bei umgekehrter Konstellation postoperativ eher niedrige Gefäßwiderstände zu erwarten sind.

Fasst man die Ergebnisse aller Studien zusammen, zeigt sich, dass die inflammatorische Antwort nach EKZ das Produkt eines komplexen Zusammenspiels humoraler und zellulärer Elemente ist. Hierbei sind im Detail nur wenige Faktoren geklärt, insbesondere auch deren zeitliche Einordnung, soll heißen die Beziehung zu vor- und nachgeschalteten Systemen. Sicher ist bisher nur, dass es durch die EKZ u. a. durch Kontakt zu Fremdoberflächen zur Aktivierung des Komplementsystems und $\mathrm{zu}$ einer Zytokinantwort kommt. Hierdurch wird eine Reaktion zwischen neutrophilen Granulozyten und Endothelzellen ausgelöst (Adhäsionsmoleküle), welche wiederum die Diapedese und Degranulation der Granulozyten mit Freisetzung von Proteasen, Sauerstoffradikalen und Arachidonsäuremetaboliten nach sich zieht. Die hieraus resultierende Gewebsschädigung führt zur Organdysfunktion. Kein anderer beschriebener Faktor (Endotoxin, NO, ET-1, PAF) konnte bis heute - unter Berücksichtigung klinischer Aspekte (Patienten-Outcome) - in einer geeigneten Studie in dieses System eingeordnet werden. Auch fehlen Studien, die prospektiv eindeutig eine Korrelation zwischen Veränderungen einzelner Laborparameter und deren Auswirkung auf den postoperativen Verlauf nachweisen.

\section{Genetische Determination der inflammatorischen Reaktion}

Es ist heute bekannt, dass genetische Faktoren eine zentrale Rolle bei der inflammatorischen Reaktion und deren klinischer Ausprägung spielen. Gen-Polymorphismen sowohl für pro- als auch antiinflammatorische Zytokine, NO-Synthase und u.a. für TNF- $\alpha$ konnten in mehreren Studien nachgewiesen werden [2]. Auch ließ sich bei erhöhten TNF-Spiegeln ein Zusammenhang zwischen bestimmten homozygoten Genkonstellationen so- wie der Entwicklung einer linksventrikulären Pumpinsuffizienz und eines Capillary-leakage-Syndroms nach EKZ zeigen. Vor diesem Hintergrund sollte bei Patienten mit einer genetisch determinierten, erhöhten proinflammatorischen Zytokinfreisetzung ein operatives Verfahren bevorzugt werden, welches EKZ und auch Ischämie-Reperfusionsschaden vermeidet (Off-pump-Verfahren).

\section{Therapieansätze}

Zur Entwicklung potenter Therapieansätze ist es unbedingt erforderlich, die komplexe Struktur der inflammatorischen Kaskade zu berücksichtigen. Es handelt sich hierbei um ein multifaktorielles Geschehen mit vielfältiger Vernetzung zwischen den einzelnen Systemen. Das Verständnis dieser Strukturen und Interaktionen ist unabdingbare Voraussetzung für eine therapeutische $\mathrm{Be}$ einflussung der Kaskade. Zahlreiche Methoden zu Unterdrückung bzw. Abschwächung der Inflammation sind in Erprobung, bisher jedoch ohne klinisch gesicherten Wirksamkeitsnachweis. Es stellt sich hier die Frage, ob es das Ziel sein muss, bei allen mittels EKZ operierten Patienten die Entzündungsantwort zu unterdrücken, oder lediglich bei jenen Patienten, bei denen diese Reaktion eskaliert. Die frühe Identifizierung dieser Patienten ist wichtig, bisher jedoch nicht zuverlässig möglich.

Zukünftig werden erfolgreiche therapeutische Strategien wesentlich von der Bestimmung des individuellen Immunstatus abhängen, d.h. von der Frage, welche Entzündungsmediatoren im jeweiligen Stadium inhibiert bzw. augmentiert werden müssen. Hierfür sind schnelle Screening-Tests erforderlich, um Patienten entsprechend ihrem individuellen Immunstatus zu therapieren. Die Entwicklung solcher, bisher nicht existenten Testverfahren muss ein Hauptziel zukünftiger klinischer Studien sein. - Tabelle 2 fasst die heute bekannten Möglichkeiten zur Beeinflussung einer systemischen Inflammation, und somit auch potenzielle Therapieansätze [13, 15, 21], zusammen. Die aktuell routinemäßig angewendeten Verfahren sind in dieser Darstellung hervorgehoben. 
Tabelle 2

Therapieansätze zur Beeinflussung der systemischen Inflammation $[13,15,21]$

\begin{tabular}{|c|c|}
\hline $\begin{array}{l}\text { Bakterien } \\
\text { (Toxine) }\end{array}$ & $\begin{array}{l}\text { - Antibiotika } \\
\text { - Antitoxine } \\
\text { - Toxinelimination (Hämofiltration [4], Plasmapherese) } \\
\text { - Immunglobuline }\end{array}$ \\
\hline humorale Systeme & $\begin{array}{l}\text { - Antikoagulation } \\
\text { - Heparinbeschichtung der EKZ-Materialien } \\
-\mathrm{C}_{1} \text {-Esterase-Inhibitoren } \\
- \text { Intraoperative Hämofiltration } \\
\text { - Kinin-Rezeptorantagonisten }\end{array}$ \\
\hline zelluläre Systeme & $\begin{array}{l}\text { - Adenosin, Xanthine } \\
\text { - Antikörper gegen Adhäsionsmoleküle } \\
\text { - Antikörper gegen Zytokine, Chemokine (TNF-a) } \\
\text { - Antikörper gegen Zytokin-Rezeptoren (TNF-a) } \\
\text { - Glukokortikoide [13] } \\
\text { - Leukozytenfilter (EKZ) }\end{array}$ \\
\hline finale Mediatoren & $\begin{array}{l}\text { Cyclooxygenaseinhibitoren } \\
\text { - Proteaseinhibitoren (Aprotinin) [11] } \\
\text { - Antioxidantien, Radikalfänger [23] } \\
\text { - PAF-Rezeptorantagonisten } \\
\text { - NO-Syntheseinhibitoren } \\
\text { - Endothelin-Rezeptorantagonisten }\end{array}$ \\
\hline
\end{tabular}

\section{Fazit}

Viele Einzelheiten der inflammatorischen Kaskade und insbesondere die jeweiligen Triggermechanismen sind bereits in zahlreichen Studien eindeutig dargestellt worden. Bis heute sind jedoch die meisten der komplexen Interaktionen zwischen den einzelnen beteiligten Systemen nur teilweise erforscht und in vielen Fällen überhaupt nicht bekannt. Die Mechanismen dieses extrem komplizierten Zusammenspiels unterliegen vielfachen Einflüssen, wie Komplementaktivierung, Endotoxinfreisetzung, Leukozytenaktivierung, Expression von Adhäsionsmolekülen und Freisetzung vieler endogener Substanzen (z. B. Sauerstoffradikale, Arachidonsäuremetabolite, Zytokine, PAF, NO und Endothelin). Es ist fraglos, dass zum Verständnis des SIRS/Sepsis-Syndroms nicht lediglich Einzelkomponenten betrachtet, sondern das gesamte System mit seinen Interaktionen berücksichtigt werden müssen, um einen Einblick in den Ablauf der inflammatorischen Kaskade zu erlangen. Auch bei der Entwicklung potenzieller Therapieansätze muss diese komplexe Vernetzung Beachtung finden. Dementsprechend wird wohl keine pharmakologische Monotherapie in der Lage sein, die destruktiven Effekte der inflammatorischen Antwort nach EKZ zu verhindern. Vielmehr muss eine Kombination gefunden werden, die entweder die Inzidenz dieser Erkrankung bzw. deren Morbidität und Letalität senkt.

In vielen herzchirurgischen Zentren kommen zu diesem Zweck Kortikosteroide und Aprotinin, sowie Heparin-beschichtete Systeme und intraoperative Ultrafiltration zum Einsatz. Der Einsatz dieser Verfahren erfolgt allerdings meist ohne gesicherte Indikation; die optimale Kombination der genannten Therapieansätze ist ebenfalls noch unklar. Aufgrund der unbefriedigenden Ergebnisse geht man heute dazu über, selektiv in die Kaskade der Inflammation einzugreifen, beispielsweise mit Antikörpern gegen Interleukine, Interleukin-Rezeptoren, TNF-a oder Adhäsionsmoleküle. Gesicherte Ergebnisse hierzu stehen noch aus; aufgrund der komplexen Vernetzung der Systeme bleibt abzuwarten, ob es mit solch selektiven Ansatzpunkten gelingt, Inzidenz und Verlauf eines SIRS zu beeinflussen.

Es ist weiterhin zu klären, in welchen Fällen die eigentlich physiologische Entzündungsreaktion nach extrakorporaler Zirkulation überhaupt therapiebedürftig ist. Wir wissen heute, dass sie bei 2 bis $10 \%$ der Patienten nach EKZ eskaliert und in großem Maße zu Morbidität und Letalität beiträgt. Es ist bis heute jedoch nicht bekannt, warum diese Eskalation mit Ausbildung eines SIRS/Sepsis-Syndroms nur bei ausgewählten Patienten abläuft. Neben der Forschung nach neuen Therapiestrategien muss deshalb die Möglichkeit der frühen Erkennung solcher „patients at risk" erstes Ziel zukünftiger Studien sein. In einer eigenen, prospektiven Untersuchung konnten wir u.a. nachweisen, dass Patienten mit einem postoperativen SIRS bereits intraoperativ (elektive ACB-Operation mit EKZ) signifikant erhöhte TNF-aSpiegel aufwiesen (- Abb. 4). Diese Beobachtung in Verbindung mit weiteren Erkenntnissen könnte dazu führen, dass spätestens intraoperativ klar sein könnte, bei welchen Patienten die Entzündungsantwort eskaliert. Da diese Information sicherlich nicht aus einem einzigen Laborparameter hervorgeht, sondern nur aus einer Kombination verschiedener Informationen, sind derlei prospektive Studien ausgesprochen aufwendig und müssen aufgrund der bekannten Inzidenz eines SIRS nach EKZ sehr viele Patienten umfassen.

\section{Literatur}

1. Boldt J, Kumle B, Papsdorf M (1998) Are circulating adhesion molecules specifically changed in cardiac surgical patients? Ann Thorac Surg 65:608-614

2. Börgermann J, Scheubel RJ, Simm A, Silber RE, Friedrich I (2007) Inflammatory response in onversus off-pump myocardial revascularization. Is ECC really the culprit ? Thorac Cardiov Surg 55:73-480

3. Galea JL, Manche A, Finn AR (1998) Circulating adhesion molecules in cardiac surgical patients [letter]. Ann Thorac Surg 66:1469

4. Groeneveld ABJ (1990) Septic shock and multiple organ failure; treatment with haemofiltration? Intensive Care Med 16:489-490

5. Hensel M, Volk T, Docke WD (1998) Hyperprocalcitonemia in patients with noninfectious SIRS and pulmonary dysfunction associated with cardiopulmonary bypass. Anesthesiology 89:93-104

6. Hess PJ (2005) Systemic inflammatory response to coronary artery bypass graft surgery. Am J Health Syst Pharm 15; 62(18 Suppl 4):6-9

7. Kalawski R, Bugajski P, Smielecki J (1998) Soluble adhesion molecules in reperfusion during coronary bypass grafting. Eur J Cardiothorac Surg 14:290-295

8. Kao CH, Hsieh KS, Wang YL (1992) The use of technetium-99m hexamethylpropylene amine oxime labelled white blood cells to detect subclinical inflammation of the heart after cardiopulmonary bypass in children with congenital heart disease. Eur J Nucl Med 19:960-963 


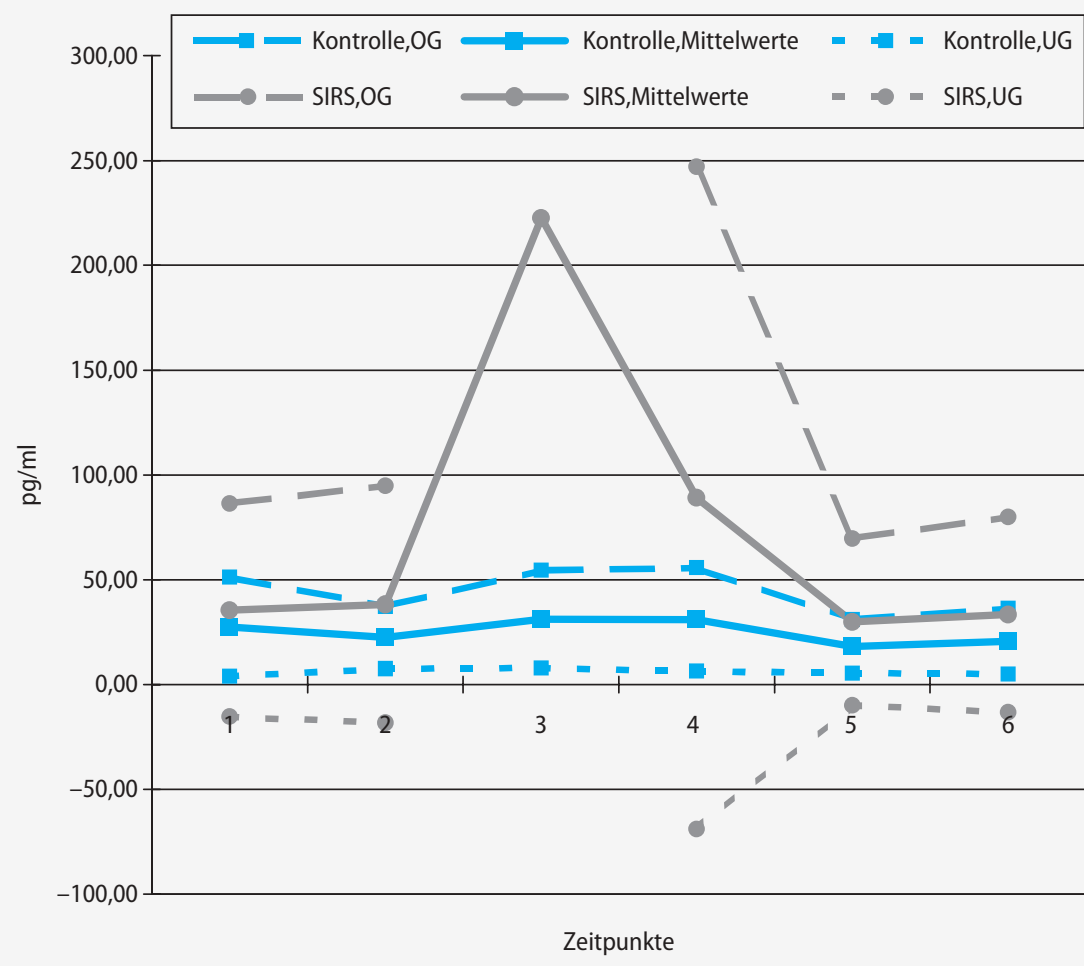

Patienten mit ACB-Operation mit EKZ;

SIRS-Gruppe $n=12$, Kontrollgruppe $n=40$;

Abnahmezeitpunkte 1: präoperativ, 2: $1 \mathrm{~h}$ nach EKZ-Beginn, 3:30 min nach EKZ-Ende,

4: Ankunft Intensivstation, 5:1. Tag po., 6:2. Tag po.

\section{Abb. 4 \ Perioperative TNFa-Verläufe für SIRS- und Kontrollgruppe (ACB-OP)}

9. Kilger E, Pichler B, Goetz AE (1998) Procalcitonin as a marker of systemic inflammation after conventional or minimally invasive coronary artery bypass grafting. Thorac Cardiovasc Surg 46:130-133

10. Koch, T, Heller S (1996) Sepsis/SIRS: Pathomechanismen und therapeutische Ansätze. Anästhesiologie und Intensivmedizin 37:386-403

11. Kubes P, Smith R, Grisham MD (1993) Neutrophilmediated proteolysis. Different roles for cathepsin Gand Elastase. Inflammation 17:321-333

12. Larmann J, Theilmeier G (2004) Inflammatory response to cardiac surgery: cardiopulmonary bypass versus non-cardiopulmonary bypass surgery. Best Pract Res Clin Anaesthesiol 18(3): 425-438
13. Lee GW, Tsou AP, Chan H (1988) Glucocorticoids selectively inhibit the transcription of the interleukin I beta gene and decrease the stability of interleukin I beta RNA. Proc Nat Acad Sci USA 85:1204-1208

14. Levy JH, Tanaka KA (2003) Inflammatory response to cardiopulmonary bypass. Ann Thorac Surg 75(2):715-720

15. Liu J, Ji B, Long C, Li C, Feng Z (2007) Comparative effectiveness of methylprednisolone and zerobalance ultrafiltration on inflammatory response after pediatric cardiopulmonary bypass. Artif Organs 31(7):571-575
16. McBride WT (2007) Mediators of inflammation at cardiac surgery. Applied Cardiopulmonary Pathophysiology 11:3-13

17. Pintar T, Collard CD (2003) The systemic inflammatory response to cardiopulmonary bypass. Anesthesiol Clin North America 21(3):453-464

18. Raja SG, Berg GA (2007) Impact of off-pump coronary artery bypass surgery on systemic inflammation: current best available evidence. J Card Surg 22(5):445-455

19. Raja SG, Dreyfus GD (2005) Modulation of systemic inflammatory response after cardiac surgery. Asian Cardiovasc Thorac Ann 13(4):382-395

20. Rastan AJ, Walther T, FalkV, Gummert JF, Eckenstein JI, Mohr FW (2006) Off-pump-Koronarrevaskularisation. Herz 31:384-395

21. Rubens FD, Mesana T (2004) The inflammatory response to cardiopulmonary bypass: a therapeutic overview. Perfusion 19 Suppl 1:5-12

22. Suleiman MS, Ascione R, Chase A, Harrington R, Angelini GD (2007) Cardiac and systemic inflammatory response during open-heart surgery with or without cardiopulmonary bypass. Applied Cardiopulmonary Pathophysiology 11:47-55

23. Suter PM, Domenighetti G, Schallert MD (1994) $\mathrm{N}$-acetylcysteine enhances recovery from acute lung injury in man. Chest 105:190-194

24. Tighe D, Moss R, Bennett D (1996) Cell surface adrenergic receptor stimulation modifies the endothelial response to SIRS. Systemic Inflammatory Response Syndrome. New Horiz 4:426-442

25. Toft P, Tonnesen E, Zulow I (1997) Expression of adhesion and activation molecules on lymphocytes during open-heart surgery with cardiopulmonary bypass. Scand Cardiovasc J 31:91-95

26. Wakiyama H, Yamashita C, Okada M (1997) Increased portal endothelin-1 level is associated with the liver function after cardiopulmonary bypass in rabbits: influence of hypothermia on the damage. Kobe J Med Sci 43:245-257

27. Wan S, LeClerc JL, Vincent JL (1997) Inflammatory response to cardiopulmonary bypass: mechanisms involved and possible therapeutic strategies. Chest 112:676-692

28. Zellner JL, Kribbs SB, Dorman BH (1998) Cardiopulmonary bypass in a gravid patient: perioperative changes in endothelin levels. Ann Thorac Surg $66: 268-270$

\section{Korrespondierender Autor}

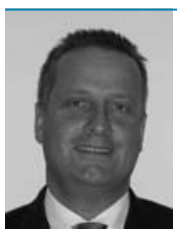

Priv.-Doz. Dr. med. Udo Boeken

Klinik für Thorax- und Kardiovaskularchirurgie

Heinrich-Heine-Universität

Moorenstraße 5, 40225 Düsseldorf, BRD

E-Mail: boeken@uni-duesseldorf.de

Dr. Udo Boeken, Jahrgang 1967, studierte von 1986 bis 1992 Humanmedizin in Düsseldorf und absolvierte auch dort seine Ausbildung zum Herzchirurgen (Facharzt 2002). Seine Habilitation erfolgte 2003. Dr. Boeken ist Weiterbildungsbefugter für chirurgische Intensivmedizin und seit 2004 Oberarzt an der Klinik für Thorax- und Kardiovaskularchirurgie der Heinrich-Heine-Universität Düsseldorf. Außerdem ist er Schriftführer der AG „EKZ und MKU“ der DGTHG.

Interessenkonflikt. Der korrespondierende Autor gibt an, dass kein Interessenkonflikt besteht. 